\title{
A hard X-ray view of giga-hertz peaked spectrum radio galaxies
}

\author{
M. Guainazzi ${ }^{1}$, A. Siemiginowska ${ }^{2}$, C. Stanghellini ${ }^{3}$, P. Grandi ${ }^{4}$, E. Piconcelli ${ }^{1}$, and C. Azubike Ugwoke \\ 1 European Space Astronomy Center of ESA, Apartado 50727, 28080 Madrid, Spain \\ e-mail: Matteo.Guainazzi@sciops.esa.int \\ 2 Harvard-Smithsonian Center for Astrophysics, 60 Garden St., Cambridge, MA 02138, USA \\ 3 Istituto di Radioastronomia, INAF, Via Gobetti 101, 40129, Bologna, Italy \\ ${ }^{4}$ Istituto di Astrofisica Spaziale e Fisica Cosmica, INAF, Via Gobetti 101, 40129, Bologna, Italy \\ 5 Enugu State University of Science and Technology, Nigeria
}

Received 5 May 2005 / Accepted 2 September 2005

\section{ABSTRACT}

We present the first broadband X-ray observations of four Giga-Hertz Peaked Spectrum (GPS) radio galaxies at redshift $\lesssim 1$ performed by $C h a n d r a$ and XMM-Newton. These observations more than double the number of members of this class with measured spectra in hard $(E>$ $2 \mathrm{keV}$ ) X-rays. All sources were detected. Their radio-to-X-ray spectral energy distributions are similar, except for PKS 0941-080, which is $\mathrm{X}$-ray under-luminous by about two orders of magnitude. The comparison between the full sample of GPS galaxies with measurements in hard $\mathrm{X}$-rays and a control sample of radio galaxies rules out intrinsic X-ray weakness as causing a lower detection rate of GPS sources in X-ray surveys. Four out of seven GPS galaxies exhibit high X-ray column densities, whereas for the remaining three this measurement is hampered by the poor spectral statistics. Bearing in mind the low number statistics in both the GPS and the control sample, the average column density measured in GPS galaxies is larger than in FR I or Broad Line Region FR II radio galaxies, but consistent with that measured in High-Excitation FR II galaxies. This leads to a location the absorbing gas in an obscuring "torus", which prevents us from observing the nuclear region along lines-of-sight perpendicular to the radio axis. This interpretation is supported by the discovery of rapid (timescale $\sim 10^{3} \mathrm{~s}$ ) X-ray variability in the GPS galaxy COINSJ0029+3456, and by an almost order-of-magnitude difference between the HI column density measured in radio and $\mathrm{X}$-rays in PKS 0500+019.

Key words. galaxies: jets - galaxies: quasars: individual: COINSJ0029+3456, PKS 0500+019, PKS 0941-080, PKS2128+048 X-ray: galaxies

\section{Introduction}

GHz Peaked Spectrum (GPS) radio sources are extremely powerful $\left(L_{\text {radio }} \gtrsim 10^{45} \mathrm{erg} \mathrm{s}^{-1}\right)$, very compact $(10-100 \mathrm{mas}$, 10-1000 parsecs) sources characterized by a simple convex radio spectrum peaking near $1 \mathrm{GHz}$ (O'Dea 1998 and references therein). GPS sources make up about $10 \%$ of the sources selected at frequencies around $5 \mathrm{GHz}$. They often exhibit symmetric structures on the parsec scale, reminiscent of those present in extended radio galaxies on much larger scales. About half of the GPS sources are classified as "GPS galaxies".

GPS sources - together with Compact Steep Spectrum (CSS) sources (O'Dea et al. 1998) - may play an important role in the history and evolution of the radio universe. It was originally suggested that they may represent radio galaxies in the early stage of their life (typical ages $<10^{4}$ years; Phillips \& Mutel 1982; Carvalho 1985; De Young 1993; Fanti et al. 1995; Readhead et al. 1996; Murgia 2003). This possibility was recently supported by the detection of micro arcseconds hotspot proper motions in a sample of $\simeq 10$ GPS galaxies (Poladitis \& Conway 2003). However, evidence for weak $\sim 10 \mathrm{kpc}$ scale radio emission in 5-10\% of all known GPS (Stanghellini et al. 1998 and references therein) suggested that the radio activity may be either recurrent (Baum et al. 1990), or smothered by the inflow of "fresh" matter after merging or interaction with a gas rich companion. Alternatively, as originally suggested by Gopal-Krishma \& Wiita (1991), GPS sources could remain compact during their radiative lifetime because interaction with dense circumnuclear matter impedes their full growth. Semi-analytical models (Carvalho 1994, 1998) and hydrodynamic simulations (De Young 1993) have indeed shown that InterStellar Medium (ISM) average densities in the range $1-10 \mathrm{~cm}^{-3}$ could be very efficient in preventing the development of large scale radio structures.

Measurements in X-rays could provide fundamental clues on the nature of GPS sources and consequently on the origin and evolution of the radio power in the universe. A hot $\left(T \sim 10^{6-7} \mathrm{~K}\right)$ and tenuous ISM phase with $n \sim$ $1 \mathrm{~cm}^{-3}$ should be a copious source of soft X-rays. However, the recent Chandra discovery of the X-ray cluster associated with the CSS quasar 3C 186 rules out the confinement of the radio source by external medium in this quasar 
(Siemiginowsha et al. 2005a). Alternatively, a distribution of cold clouds would imprint an X-ray photoelectric cutoff on the soft X-ray spectrum. It has been noted that GPS sources are rather elusive in X-rays. In the ROSAT All Sky Survey, GPS/CSS quasars exhibited a detection rate three times lower than radio-loud quasars of comparable power (Baker et al. 1995). Whether this low detection rate is due to intrinsic weakness or to obscuration of the active nucleus is still largely unknown. Prior to the launch of Chandra and XMM-Newton, high-quality CCD-resolution hard $(E>2 \mathrm{keV}) \mathrm{X}$-ray spectra were available for two GPS galaxies only: NGC 1052 (Guainazzi et al. 2000) ${ }^{1}$ and 4C+12.50 (O’Dea et al. 2000). Both exhibit a high X-ray obscuring column density $\left(N_{\mathrm{H}}>\right.$ $10^{22} \mathrm{~cm}^{-2}$ ).

In order to gain insight into the X-ray under-luminosity of GPS galaxies, we have performed Chandra and XMM-Newton observations of 5 GPS galaxies at $z \lesssim 1$. This paper presents the results of this program. The observations discussed here represent the first hard X-ray measurements of the target sources. They more than double the number of GPS galaxies with hard X-ray measurements. The results of the observation of Mkn $668(\mathrm{OQ}+208)$, performed in the framework of our program, were presented by Guainazzi et al. (2004). This observation allowed the discovery of the first radio-loud Comptonthick $\left(N_{\mathrm{H}}>\sigma_{t}^{-1}\right)$ AGN.

Unless otherwise specified: energies are quoted in the source reference frame; uncertainties on the spectral parameters are at the $90 \%$ confidence level for one interesting parameter; upper limits are also at the $90 \%$ confidence level; other uncertainties are at the $1 \sigma$ level; in the calculation of the luminosities, we adopted a Hubble constant of $70 \mathrm{~km} \mathrm{~s}^{-1} \mathrm{Mpc}^{-1}$ (Bennett et al. 2003); data points in the spectral plots correspond to a signal-to-noise $>3$. We have used throughout the paper the Gehrels algorithm (Gehrels 1986) to estimate the statistical uncertainties associated with Poissonian-distributed measurements.

\section{The sample}

In this section we present a short description of the radio properties of the sources discussed in this paper, supporting their classification as "GPS" galaxies. We require both a convex radio spectrum and a symmetric radio morphology to classify a radio galaxy as GPS.

COINS J0029+3456 (B 0026+346) is a galaxy with $m_{r}=21.0^{2}$ at $z=0.517$ (Snellen et al. 1996; Zensus et al. 2002). It has a broad radio spectrum, which is rather flat at high frequencies (Kühr et al. 1981). It exhibits a compact symmetric morphology with an overall size of 35 mas (190 pc).

$P K S 0500+019(\mathrm{~J} 0503+0203)$ is a radio galaxy with $m_{i}=21.0$ at $z=0.583$ (Carilli et al. 1998) with a Compact Symmetric Object (CSO) morphology which extends for about 15 mas

\footnotetext{
${ }^{1}$ See Sect. 5.2 for a discussion on the GPS classification of this radio galaxy.

${ }^{2} m_{r}$ and $m_{i}$ are the magnitudes in the $r$ and $i$ band, respectively, according to the Gunn system.
}

(84 pc). Stickel et al. (1996) reported an emission line at a higher redshift, therefore they consider the GPS radio source associated with a background quasar and the galaxy as an intervening object. De Vries et al. (2000), however, did not find any trace of this emission line in their optical spectra.

PKS 0941-080 is a radio galaxy at $z=0.228$ with a clear convex radio spectrum and a rather clear CSO morphology (Dallacasa et al. 1997).

PKS 2128+048 (J2130+0502) is a galaxy with $m_{r}=23.3$ at $z=0.99$ (Snellen et al. 1996) with a clear GPS radio spectrum and a rather clear CSO morphology of 35 mas (220 pc) (Stanghellini et al. 1997, 2001).

Table 1 reports the list of observations discussed in this paper. The March 2004 observation of PKS 0500+019 was heavily affected by high-particle background, which reduced the usable exposure time to $\simeq 2 \mathrm{ks}$. The target was therefore successfully re-observed in August.

\section{XMM-Newton results}

The XMM-Newton data were reduced with SAS v6.0 (Gabriel et al. 2003), using the most updated calibration files (January 2005). In this paper, only data from the EPIC cameras (MOS; Turner et al. 2001; pn, Strüder et al. 2001) will be discussed. Event lists from the two MOS cameras were merged before accumulation of any scientific products. Single to double (quadruple) events were used to accumulate pn (MOS) spectra. High-background particle flares were removed by applying fixed thresholds on the single-event, $E>10 \mathrm{keV}$, $\Delta t=10 \mathrm{~s}$ light curves. These thresholds, as well as the radius of the source scientific product circular extraction regions, were optimized to maximize the signal-to-noise ratio, and are listed in Table 1. Background scientific products were extracted from annuli around the source for the MOS, and from circular regions in the same chip for the pn, at the same height in detector coordinate as the source location. Spectra were binned in order to oversample the intrinsic instrumental energy resolution by a factor $\geq 3$, and to have at least 25 counts in each background-subtracted spectral channel. This ensures that the $\chi^{2}$ statistics can be used to evaluate the quality of the spectral fitting. pn (MOS) spectra were fitted in the $0.35-15 \mathrm{keV}$ $(0.5-10 \mathrm{keV})$ spectral range.

\subsection{Source identification}

In Table 2 we list the coordinates of the nominal targets in our XMM-Newton observations. They were determined on the $0.5-10 \mathrm{keV}$ image extracted from merged event lists of the three EPIC cameras. Typical statistical errors are $\simeq 1^{\prime \prime}$, whereas typical systematic uncertainties on the absolute attitude reconstruction are $\simeq 1.5^{\prime \prime}$. For PKS $0500+019$ (2nd observation) the X-ray position is shifted by about $4.5^{\prime \prime}$ in RA with respect to the position of the radio source (Condon et al. 1998; Ma et al. 1998), corresponding to about $25 \mathrm{kpc}$ at the source redshift. This difference exceeds the expected measurement uncertainties. The XMM-Newton position agrees well with that of the closest source in the ROSAT PSPC catalog (Voges et al. 2000). No optical counterpart exists for either the X-ray or the 
Table 1. Log of the observations discussed in this paper. "Threshold" indicates the maximum background count rate used in the generation of appropriate time intervals for the extraction of spectra. The "Radius" refers to the source extraction region.

\begin{tabular}{lcccccc}
\hline \hline Source & $z$ & $\begin{array}{c}N_{\mathrm{H}, \mathrm{Gal}} \\
\left(10^{20} \mathrm{~cm}^{-2}\right)\end{array}$ & Start date & $\begin{array}{c}T_{\exp }{ }^{a} \\
(\mathrm{ks})\end{array}$ & Threshold $\left(\mathrm{s}^{-1}\right)$ & ${\text { Radius }\left({ }^{\prime \prime}\right)^{a}}$ \\
\hline COINS J0029+3456 & 0.571 & 5.6 & 08-Jan.-2004 & $12.2 / 10.8$ & $0.25 / 1.0$ & $35 / 40$ \\
PKS 0500+019 (1) & 0.584 & 8.3 & 09-Mar.-2004 & $2.0 / 1.9$ & $2.0 / 1.0$ & $20 / 30$ \\
PKS 0500+019 (2) & & & 18-Aug.-2004 & $9.6 / 7.2$ & $0.0 / 0.0$ & $20 / 30$ \\
PKS 0941-080 & 0.228 & 3.7 & 26-Mar.-2002 & 5.3 & $\ldots$ & 1.4 \\
PKS 2128+048 & 0.990 & 5.2 & 11-Oct.-2002 & 5.7 & $\ldots$ & 2.4 \\
\hline
\end{tabular}

${ }^{a}$ For XMM-Newton MOS/pn and Chandra ACIS, respectively.

Table 2. GPS sample sources X-ray coordinates. " $d$ " indicates the distance between the X-ray and the radio coordinates, the latter extracted from the NED catalogue. "CTS" are net counts in the 0.5-10 keV and $0.1-11 \mathrm{keV}$ energy bands for the XMM-Newton EPIC and the Chandra ACIS, respectively. The values for PKS 0500+019 refer to the second observation only.

\begin{tabular}{ccccc}
\hline \hline Source & $\begin{array}{c}\text { RA } \\
(\mathrm{J} 2000)\end{array}$ & $\begin{array}{c}\text { Dec } \\
(\mathrm{J} 2000)\end{array}$ & $d\left(^{\prime \prime}\right)$ & CTS \\
\hline 0029 & $00^{\mathrm{h}} 29^{\mathrm{m}} 14^{\mathrm{s}} .1$ & $+34^{\circ} 56^{\prime} 32^{\prime \prime}$ & 1.7 & $1030 \pm 40$ \\
0500 & $05^{\mathrm{h}} 03^{\mathrm{m}} 20^{\mathrm{s}} .9$ & $+02^{\circ} 03^{\prime} 05^{\prime \prime}$ & 4.5 & $1350 \pm 40$ \\
0941 & $09^{\mathrm{h}} 43^{\mathrm{m}} 36^{\mathrm{s}} .9^{a}$ & $-08^{\circ} 19^{\prime} 31^{\prime \prime} a$ & $\ldots$ & $10 \pm 3$ \\
2128 & $21^{\mathrm{h}} 30^{\mathrm{m}} 32^{\mathrm{s}} .8$ & $+05^{\circ} 02^{\prime} 08^{\prime \prime}$ & 1.1 & $92 \pm 10$ \\
\hline
\end{tabular}

${ }^{a}$ Fixed to the radio position.

the radio position. The paucity of X-ray/optical coincidences in the XMM-Newton field prevented us from performing an independent calibration of the absolute astrometry in this observation. Nonetheless, a comparable shift in RA is observed between several XMM-Newton sources and the closest Digital Sky Survey plate source. Although this evidence does not constitute proof, we will consider in the following such a shift as due to residual inaccuracies in the attitude reconstruction.

\subsection{Timing analysis in COINS J0029+3456}

The light curves observed during the XMM-Newton observations are consistent with being constant in all energy ranges, except for COINS J0029+3456, where variability of a factor of $\simeq 3$ within a few thousand seconds was observed (Fig. 1). By fitting consecutive segments of the light curve with a linear function, we estimate a typical luminosity change rate of $(5.0 \pm 2.5) \times 10^{43} \mathrm{erg} \mathrm{s}^{-1}$ hour $^{-1}$. We find only marginal evidence that the variability is primarily due to hard X-rays. The reduced $\chi^{2}$ resulting from fitting with a constant line the $\Delta t=1024 \mathrm{~s}$ binned light curves is 0.86 and 1.27 in the $0.2-1$ and 1-10 keV energy band, respectively. Consequently, no significant variability of the hardness ratio is associated with these flux changes. We will therefore discuss the time-averaged spectrum of this source in Sect. 3.3. COINS J0029+3456 also shows a remarkable historical variability in the soft X-ray band. The $1 \mathrm{keV}$ flux density decreased from $100 \pm 30 \mathrm{nJy}$ (Einstein/IPC, 1980) and $63 \pm 15$ nJy (ROSAT/PSPC, 1992)

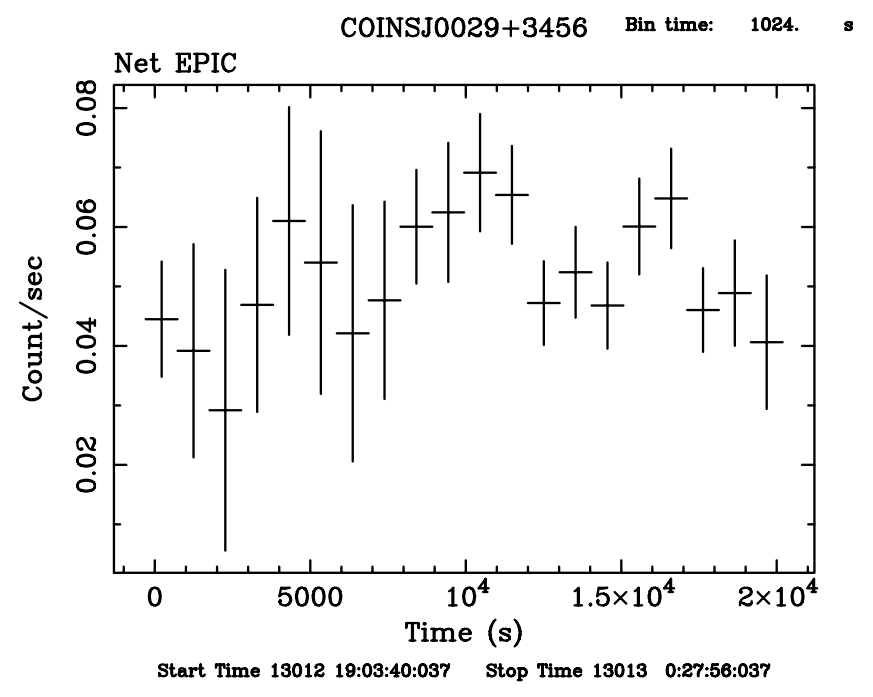

Fig. 1. $0.5-10 \mathrm{keV}$ EPIC (pn and MOS) light curve during the XMM-Newton observation of COINS J0029+3456. The binning time is $\Delta t=1024 \mathrm{~s}$. Times are in seconds after the observation start.

(Kollgaard et al. 1995; our re-analysis of archival data) to $29 \pm_{7}^{9} \mathrm{nJy}$ as measured about 10 years later (January 2004) by XMM-Newton/EPIC. It is impossible to tell whether a change of the column density of the intervening absorber contributes to this variability, due to the lack of statistical quality and energy resolution of the measurements prior to XMM-Newton.

\subsection{Spectral analysis}

Spectra of the EPIC cameras have been simultaneously fitted, allowing only a free cross-normalization factor between them. We have applied a "baseline model", constituted by a powerlaw modified by photoelectric absorption. We have modeled the absorption simultaneously with two components: a) the intervening gas along the line of sight in our Galaxy, with a column density $N_{\mathrm{H}, \mathrm{Gal}}$ held fixed to the value determined by the $21 \mathrm{~cm}$ radio maps (Dickey \& Lockman 1990) and; b) a "local" absorber located at the same redshift as the systemic velocity of each galaxy; its column density, $N_{\mathrm{H}}$, was left as a free parameter in the fit. All the continuum parameters were left free in the fit as well. This baseline model yields a good fit for all observations. The spectral parameters derived from the two observations of PKS $0500+019$ are formally consistent within the 
Table 3. Spectral results on the XMM-Newton GPS sample described in this paper. The model employed to fit the data is a photoelectrically absorbed power-law. The obscuring gas with column density $N_{\mathrm{H}}$ is assumed to be located at the systemic velocity of the galaxy. $\Gamma$ is the photon index of the power-law, $F$ the observed flux, $L$ the intrinsic luminosity, corrected for absorption.

\begin{tabular}{lccccc}
\hline \hline Source & $\begin{array}{c}N_{\mathrm{H}} \\
\left(10^{21} \mathrm{~cm}^{-2}\right)\end{array}$ & $\Gamma$ & $F^{a}$ & $L^{b}$ & $\chi^{2} / v$ \\
\hline COINSJ 0029+3456 & $10 \pm_{4}^{5}$ & $1.43 \pm_{0.19}^{0.20}$ & 2.7 & $2.3 \pm 0.2$ & $38.9 / 34$ \\
PKS 0500+019 (1) & $10 \pm_{9}^{11}$ & $2.0 \pm_{0.5}^{0.7}$ & 3.9 & $3.8 \pm 0.9$ & $10.3 / 9$ \\
PKS 0500+019 (2) & $5 \pm_{2}^{3}$ & $1.62 \pm_{0.19}^{0.21}$ & 5.6 & $5.0 \pm 0.6$ & $33.1 / 44$ \\
PKS 0941-080 & $\ldots$ & $2^{c}$ & 0.07 & $0.009 \pm 0.008$ & $\ldots{ }^{d}$ \\
PKS 2128+048 & $3.0 \pm_{3.0}^{8.1}$ & $1.5 \pm_{0.7}^{0.6}$ & 1.6 & $4.4 \pm 1.1$ & $1.7 / 4$ \\
${ }^{a}$ In units of $10^{-13} \mathrm{erg} \mathrm{cm}^{-2} \mathrm{~s}^{-1}$ in the $0.5-10 \mathrm{keV}$ energy band (observer's frame). \\
${ }^{b}$ In units of $10^{44} \mathrm{erg} \mathrm{s}^{-1}$ in the 2-10 keV band (source frame), corrected for absorption. \\
${ }^{c}$ Fixed. \\
${ }^{d}$ Not enough independent channels for a spectral fit to be possible.
\end{tabular}
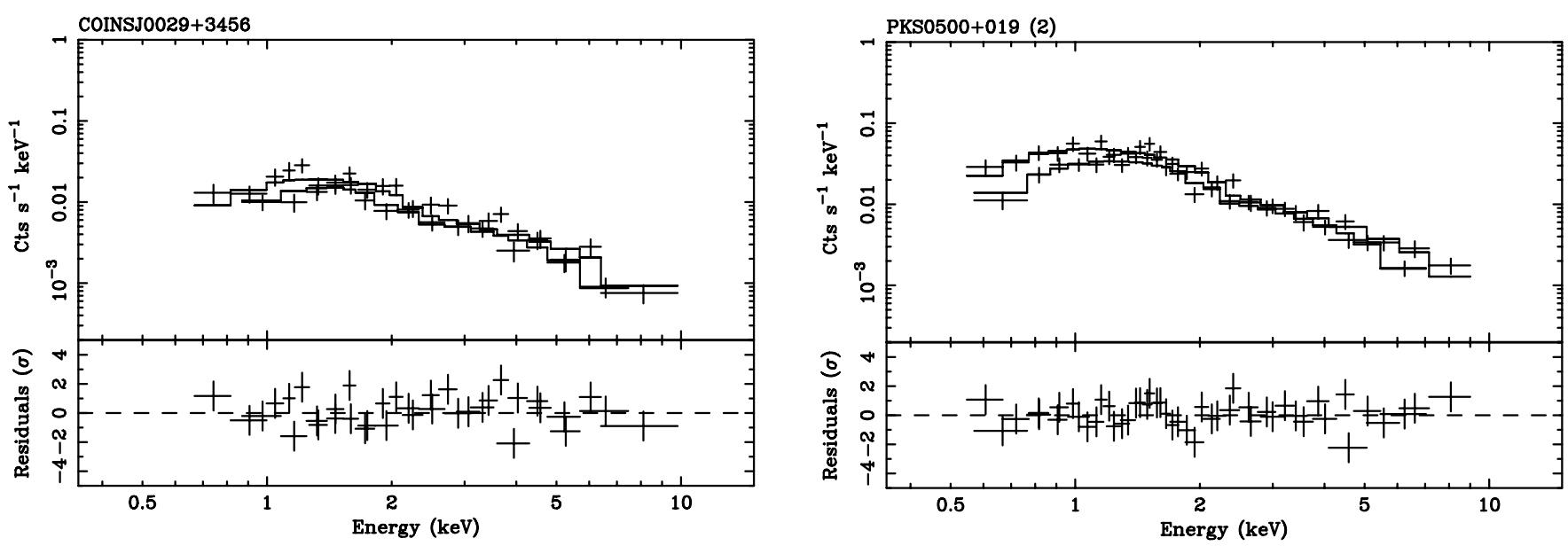

Fig. 2. Spectra (upper panels) and residuals in units of standard deviations (lower panels) when the best fit model of Table 3 is applied to the XMM-Newton/EPIC spectra of COINS J0029+3456 and PKS 0500+019.

statistical uncertainties, except for $\mathrm{a} \simeq 40 \%$ larger average flux in the later one. We will consider in the following the results obtained during the second observation of PKS 0500+019, as their associated statistical uncertainties are smaller.

Table 3 summarizes the spectral parameters of the XMM-Newton GPS sample presented in this paper. The spectra and residuals against the best fit models are shown in Fig. 2.

\section{Chandra results}

Two GPS galaxies, PKS 0941-080 and PKS 2128+048, were observed with the Chandra Advanced CCD Imaging Spectrometer (ACIS-S, Weisskopf et al. 2002) as part of a program to observe a sample of GPS sources (Siemiginowska et al. 2005b). The short $5 \mathrm{ksec}$ observations were mainly scheduled to measure the X-ray flux. The sources were located $\sim 35^{\prime \prime}$ from the default aim-point position (to avoid node boundaries) on the ACIS-S backside illuminated chip S3 (Proposer's Observatory
Guide $\left.^{3}\right)$. The $1 / 8$ sub-array CCD readout mode of one CCD only was used resulting in a $0.441 \mathrm{~s}$ frame readout time.

The X-ray data analysis was performed in CIAO $3.1^{4}$ with the calibration files from the CALDB 2.28 data base. Note that the ACIS-S contamination file acisD1999-08-13contamN0003.fits which incorporates effects of the ACIS-S contamination into the effective area was included in our analysis. The spectra were rebinned according to the same criteria employed for XMM-Newton (see Sect. 3).

The two galaxies were detected by Chandra with the count rates listed in Table 2. For PKS 0941-080 the total number of net counts $(\simeq 10)$ is too low to perform a true spectral fitting. In PKS $2128+048$ a simple power-law model with photoelectric absorption yields a good fit. A column density of $N_{\mathrm{H}} \simeq 3 \times 10^{21} \mathrm{~cm}^{-2}$ is measured. However this measurement is still consistent with 0 if the large statistical uncertainties are taken into account.

\footnotetext{
${ }^{3}$ http://asc.harvard.edu/proposer/POG/index.html

${ }^{4}$ http://cxc.harvard.edu/ciao/
} 

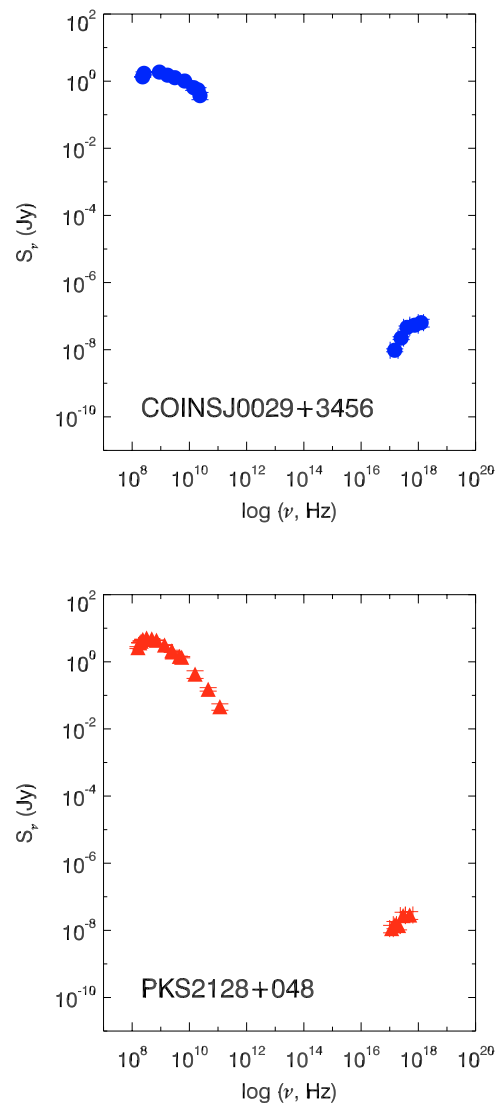
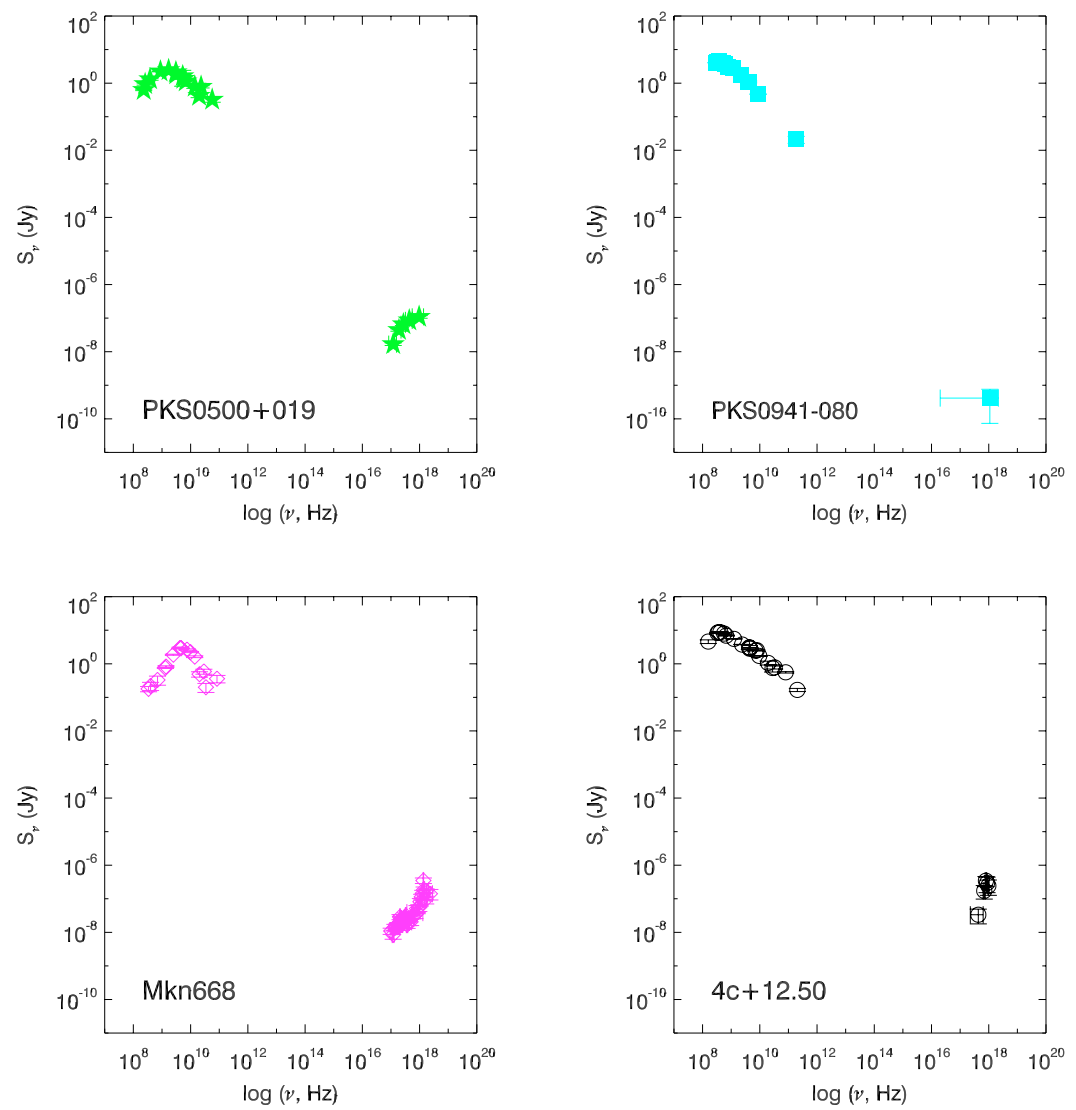

Fig. 3. Radio-to-X-ray Spectral Energy Distributions for the objects of our sample, 4C+12.50 (O'Dea et al. 2000) and Mkn 668 (Guainazzi et al. 2004). Radio measurements represent not simultaneous observations compiled from the NASA Extragalactic Database (NED).

\section{Discussion}

\subsection{Spectral Energy Distributions}

In Fig. 3 we show the radio-to-X-ray Spectral Energy Distributions (SEDs) for the GPS galaxies discussed in this paper, as well as for 4C+12.50 (O’Dea et al. 2000) and Mkn 668 (Guainazzi et al. 2004). The $5 \mathrm{GHz}$ to $5 \mathrm{keV}$ flux density ratios of 5 out of 6 objects are within a factor of 15 . On the other hand, in PKS $0941+080$ the $5 \mathrm{GHz}$ to $5 \mathrm{keV}$ flux density ratio is about two orders-of-magnitude lower than the average for the rest of the sample. Whether this is due to intrinsic weakness or extreme (i.e. Compton-thick) obscuration is impossible to tell with the current data, and would require very deep X-ray exposures to be finally elucidated. There is no correlation between the X-ray to radio flux density ratio and the radio break frequency; however, an anti-correlation at the $2 \sigma$ level is found between the X-ray to radio flux density ratio and the radio spectral index above the frequency break (Stanghellini et al. 1998; see Fig. 4).

Mkn 668 is a peculiar object in our small sample. Not only does it exhibit the largest measured obscuration among the GPS galaxies for which hard X-ray measurements are available (being one of the very few known radio-loud Compton-thick AGN), it also shows a prominent soft X-ray excess above the extrapolation of the obscured primary AGN continuum. None of the other GPS exhibits this component in the XMM-Newton or Chandra spectra. Its origin is still to be fully elucidated, the most likely possibilities being scattering of the AGN radiation or inverse-Compton recoil of the large far infrared emission (Mazzarella et al. 1991; Guainazzi et al. 2004).

\subsection{Comparison with a control sample of "normal" radio galaxies}

In this section we compare the spectral properties of the full sample of GPS galaxies for which hard X-ray measurements are available with a control sample of Radio Galaxies (RGs). The former includes, with the objects discussed in this paper: 4C+12.50 (O'Dea et al. 2000), 4C+55.16 (Iwasawa et al. 1999), OQ+208 (Guainazzi et al. 2004). We do not include the radio galaxy NGC 1052, despite it being sometimes classified as a "recurrent" GPS source (Tingay et al. 2003; Edwards \& Tringay 2004). NGC 1052 has a 2 -sided jet morphology at the mas scale, without evidence of micro hot spots (Kadler et al. 2004; Satypal et al. 2004). The hot spots are instead present at large scales, implying that the the jets are a continuous structure, which ends at the kpc scale.

The control sample is taken from a compilation of radioloud AGN X-ray spectra discussed by Sambruna et al. (ASCA, 1999; S99 hereafter), Donato et al. (Chandra, 2004; D04), and Fiocchi et al. (BeppoSAX, 2005; F05). S99 provides in the redshift range of the GPS sample a not statistically complete sample of 15 radio galaxies of mixed FR I and FR II types, and optical (broad/narrow line) properties. To this, F05 add five more 


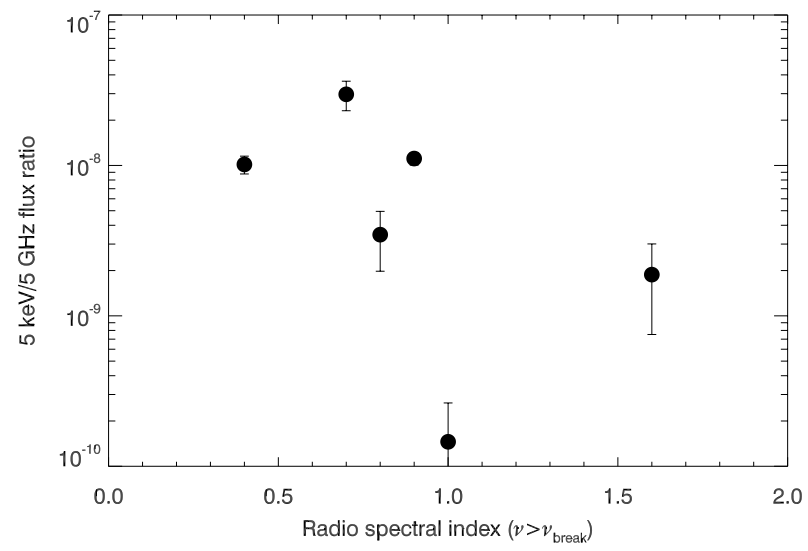

Fig. 4. $5 \mathrm{keV}$ to $5 \mathrm{GHz}$ flux density ratio as a function of the radio spectral index above the break frequency. The slope of a linear least squares fit is $(-1.2 \pm 0.6) \times 10^{-8}$. The slope does not significantly change if the data point corresponding to PKS 0941+080 is excluded from the fit.

FR II and D04 three more FR I galaxies, again of mixed optical types. This comparison aims to address the following basic questions: a) are GPS galaxies intrinsically X-ray weaker than "normal" radio galaxies?; b) are GPS galaxies more obscured than "normal" radio galaxies?

\subsubsection{Are GPS galaxies intrinsically X-ray weaker than "normal" radio galaxies?}

No. In Fig. 5 we show the location of the GPS sample and of the control radio galaxy sample in the $5 \mathrm{GHz}$ versus $2-10 \mathrm{keV}$ luminosity plane (we stress that the X-ray luminosity is intrinsic, i.e. corrected for absorption). GPS galaxies occupy the same region of the $L_{\mathrm{X}}$ versus $L_{\text {radio }}$ plane as "normal" radio galaxies across the whole range of $\mathrm{X}$-ray luminosities covered by the sample. The average luminosity ratios $R_{\mathrm{Xr}}$ are: $\log \left[R_{\mathrm{Xr}}(\mathrm{GPS})\right]=0.7 \pm 0.3, \log \left[R_{\mathrm{Xr}}(\mathrm{RG})\right]=0.69 \pm 0.17$.

The origin of the X-ray continuum in GPS galaxies is still largely unknown. Observed spectral indices are in the range 1.4-2.0, therefore consistent with both accretion- (Perola et al. 2002; Risaliti 2002) or beamed-dominated (Fossati et al. 1998) systems. The upper limits on the equivalent width of the $\mathrm{K}_{\alpha}$ fluorescent iron line $(\$ 150 \mathrm{eV})$ are not stringent enough to rule out the former explanation. Moreover, for none of the objects presented in this paper are measurements at $7000 \AA$ available, which may contribute to elucidate the nature of the central core continuum through the radio-to-UV correlation in Chiaberge et al. (1999).

\subsubsection{Are GPS galaxies more obscured than "normal" radio galaxies?}

This may be true. In a recent Chandra survey of FR I RGs (D04), only one out of 15 objects exhibit a column density $\simeq 10^{22} \mathrm{~cm}^{-2}$, whereas for 13 of them $N_{\mathrm{H}} \leq 10^{21.3} \mathrm{~cm}^{-2}$ (D04). In Fig. 6 we show the measured column densities for the GPS sample and the FR II sample of S99 and F05, together with the $N_{\mathrm{H}}$ distribution function for the two classes. The apparent

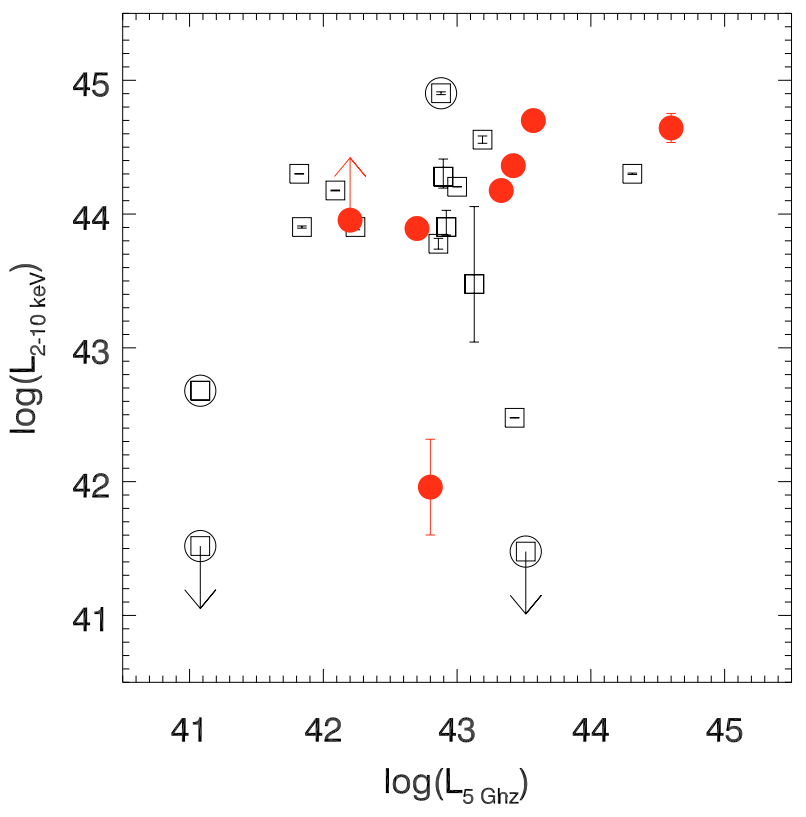

Fig. 5. $5 \mathrm{GHz}$ versus $2-10 \mathrm{keV}$ for the control sample radio galaxies (empty squares) and the GPS galaxies sample (filled circles). Empty circles surround the FR I radio galaxies data points.

anti-correlation between $N_{\mathrm{H}}$ and $z$ is most likely due to a selection effect, as farther objects with greater obscuration are more easily missed. All the measurements of $N_{\mathrm{H}}$ in GPS galaxies lie on the upper envelope of the plot. With the caveat of the small numbers, the two distributions are different at the $98.3 \%$ level, according to the Kolmogorov-Smirnov (K-S) test. The difference is primarily due to the relative lack of unabsorbed GPS galaxies. Only one out of five GPS galaxies $(<46 \%)$ has $N_{\mathrm{H}} \lesssim 10^{22} \mathrm{~cm}^{-2}$, compared to $75 \pm 26 \%$ RGs. If the Xray weakness of PKS 0941-080 is due to high obscuration, or we include in the sample NGC $1052\left(N_{\mathrm{H}} \simeq 2 \times 10^{23} \mathrm{~cm}^{-2}\right.$, Guainazzi et al. 2000) the evidence for greater absorption in GPS galaxy is even more significant (see as well Guainazzi et al. 2004). These results are still sensitive to Poissonian noise. Their confirmation on a larger sample is a task we are actively pursuing.

Neither the control sample nor the GPS sample are, by construction, unbiased or complete. However, they are reasonably well matched in both radio luminosity and redshift. The K-S probability that the radio luminosity distributions for GPS and the control sample are drawn from the same parent population is $66.5 \%$. The same probability for the redshift distribution is somewhat smaller (37.4\%; cf. Fig. 7). This is potentially more worrying, as the GPS redshift distribution median is larger than in the control sample, and obscured objects are in principle more easily detected at higher redshifts. In order to estimate the potential systematic error due to comparing column density distributions corresponding to samples with different redshift distributions, we have simulated a large number of spectra, using the best-fit models applicable to the GPS objects of our sample, but assuming that they are distributed in redshift in the same way as the objects of the control sample. The difference between the observed and the simulated GPS $N_{\mathrm{H}}$ 

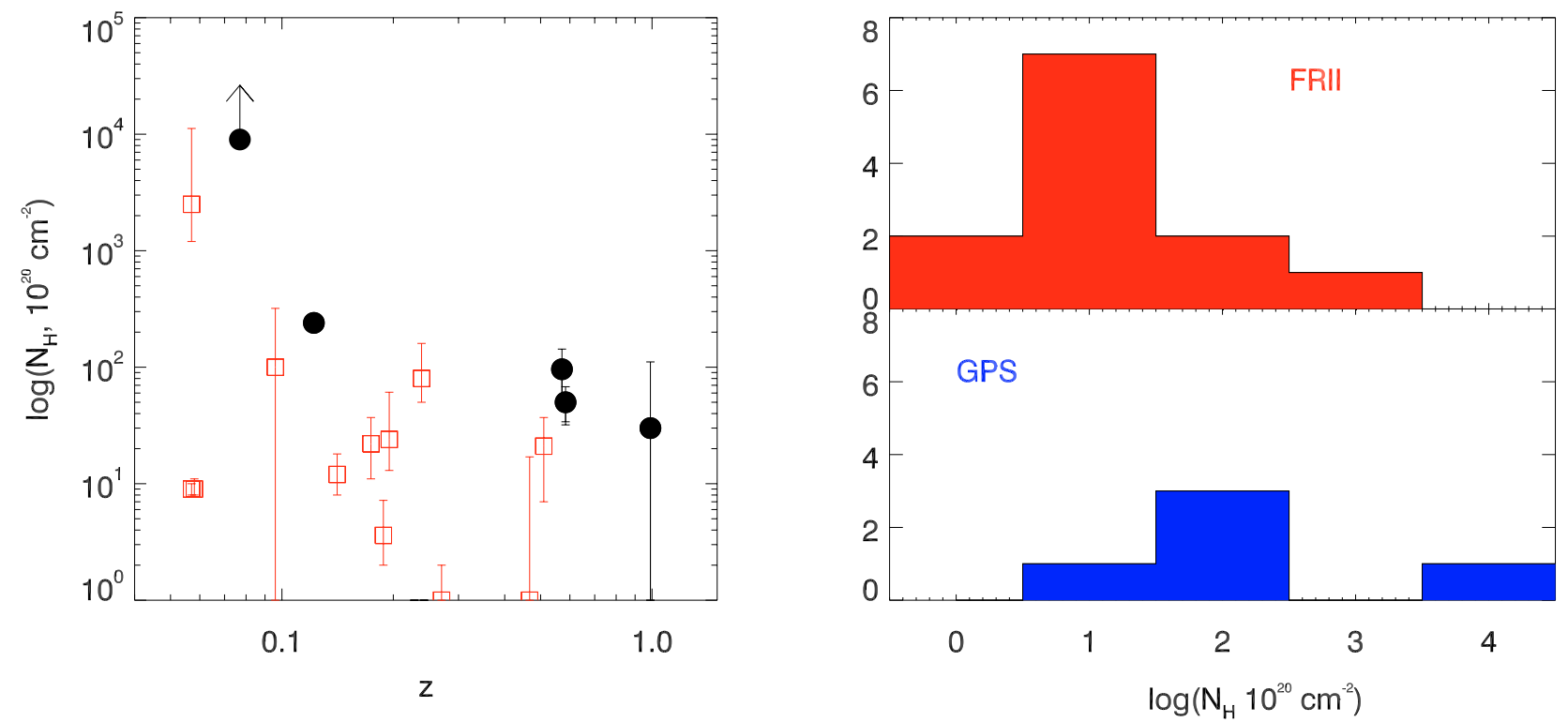

Fig. 6. Left panel: $N_{\mathrm{H}}$ versus $z$ distributions for GPS galaxies (filled circles) and the control sample of FR II radio galaxies (empty squares). Right panel: the $N_{\mathrm{H}}$ distribution functions for FR II radio galaxies (top) and GPS galaxies.

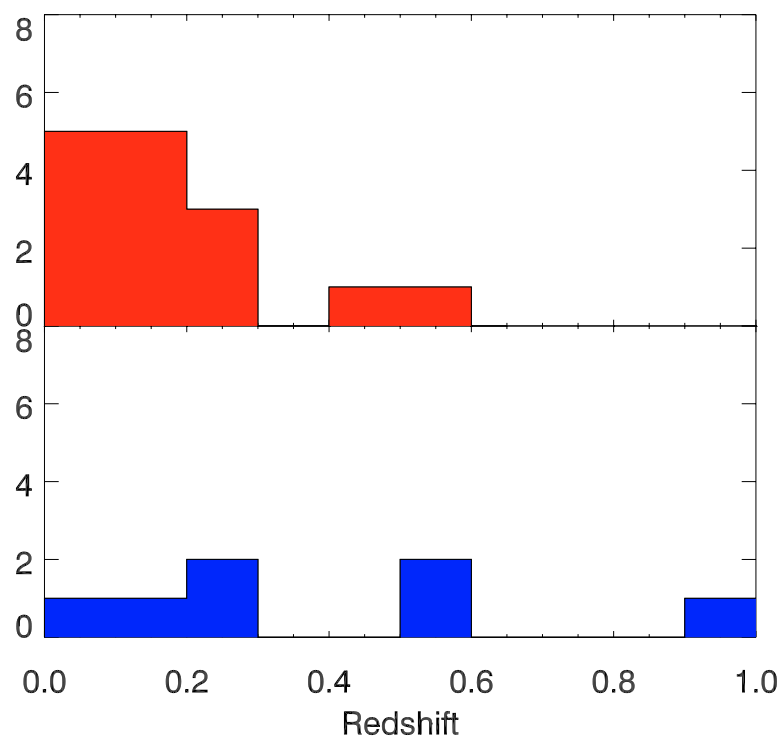

Fig. 7. Redshift distribution for the GPS sample (lower panel) and the "normal" radio galaxy control sample (upper panel).

distributions were parameterized through the statistical uncertainty-weighted average of the shift in the column densities, $\Delta N_{\mathrm{H}}$. The shifts are $-0.19 \pm 0.09 \mathrm{dex}$ and $-0.08 \pm 0.09 \mathrm{dex}$ only, when the redshift distributions for FR II and FR I are used.

Previous studies in the optical and UV revealed a high detection rate for FR I RGs (Chiaberge et al. 1999, 2002). Their X-ray spectra are generally unobscured (D04) The emission of their central compact core has been interpreted as non-thermal synchrotron radiation from the base of the jet, suggesting that the we are observing the innermost regions close to the supermassive black hole. In the framework of the grand-unification scenario for FR sources (Urry \& Padovani 1995) the larger obscuration of GPS galaxies could be ascribed to their orientation with respect to an azimuthally-symmetric absorbing structure ("torus" hereafter), which prevents us from directly observing the central regions in objects observed along lines-of-sight perpendicular to the jet axis.

More puzzling is the evidence that GPS galaxies are "more obscured" than FR II objects as well. According to a classification originally due to Jackson \& Rawlings (1997), FR IIs can be distinguished among Broad Line Objects (BLO), and High- and Low-Excitation galaxies (HEG, LEG; Laing et al. 1994). BLOs are interpreted as unobscured nuclei (Varano et al. 2004). Optical nuclei of LEGs are detected in a comparable fraction as FR I, and are therefore more similar to that class (which requires some revision of the standard zero-th order unification scenario). Finally, 50\% of the HEG do not show any optical counterpart, and should represent the "true" torusobscured radio-loud AGN population. The covering fraction of this "torus" seems to be redshift-dependent, its opening angle widening up as the accretion disk luminosity increases (Varano et al. 2004; see as well Page et al. 2003; Jiménez-Bailón et al. 2005). In our control sample, both BLO (5 objects) and LEG (2) FR II RGs exhibit typically $N_{\mathrm{H}} \lesssim 3 \times 10^{21} \mathrm{~cm}^{-2}$. The HEG (4) show a much larger spread in column densities $\left(N_{\mathrm{H}} \lesssim 10^{23} \mathrm{~cm}^{-2}\right)$, with a $\left\langle\log \left(N_{\mathrm{H}, \mathrm{HEG}}\right)\right\rangle=2.7 \pm 0.6$, consistent with that of the GPS galaxy sample [ $\left.\left\langle\log \left(N_{\mathrm{H}, \mathrm{GPS}}\right)\right\rangle \geq 2.2 \pm 0.7\right]$. At face value, this implies that the line-of-sight to HEG FR II and GPS galaxies AGN intercepts matter of comparable density, suggesting a common orientation with respect to the obscuring "torus" postulated for the former. No measurement of the orientation of the radio structure is available for the objects of the GPS sample. However, their symmetric morphology suggests that the inclination angle is not small. If X-rays originate close to the supermassive black hole, and a torus surrounds the central region, our line-of-sight will very likely intercept it.

Most of the GPS host galaxies in our sample exhibit a distorted morphology (4C+12.50, PKS 0500+019, Mkn 668) and/or double nucleus or close companions (all except 
COINS J0029+3456, Peacock et al. 1981; Biretta et al. 1985; Fugmann \& Meisenheimer 1988; Stanghellini et al. 1993; de Vries et al. 1995; Stickel et al. 1996). High-resolution $2.2 \mu$ NICMOS/HST images of 4C+12.50 and PKS 0941-080 confirm the presence of double nuclei and, in the former case, of a highly disturbed morphology (Stanghellini et al., submitted; Guainazzi et al., in preparation; see as well de Vries et al. 1998, 2000 for ground-based near-infrared observations). These reports indicate recent mergers. The role of gravitational torques induced by galaxy mergers in driving gas inflows toward the nucleus has been highlighted by numerical simulations (Barnes \& Hernquist 1996; Taniguchi \& Wada 1996). Indeed, interacting AGN pairs seem to invariably host X-ray obscured nuclei (see Guainazzi et al. 2005, and references therein). Galaxy mergers could therefore represent the link between the large amount of gas in the GPS nuclear environment and the disturbed and multiple optical morphologies of GPS host galaxies (O'Dea et al. 1996).

Does the gas probed by X-ray measurements have enough density to influence the evolution of the parsec-scale jet? Guainazzi et al. (2004) demonstrated in the cases of Mkn 668 that if the parsec-scale jet had to pierce its way through the Compton-thick absorber covering the innermost nuclear region, this could lead to an underestimate by up to two orders of magnitude of the jet dynamical age as derived from the hotspot recession velocity. Even a Compton-thick absorber would not be enough to ultimately "choke" the expanding jet. However, measurements of HI absorbing gas (Pihlström et al. 2003) suggest a particle density of $n_{\mathrm{e}} \simeq 30 \mathrm{~cm}^{-3}$ at the external surface of the "drilling jet" (consistent with a residual column density covering the soft X-ray excess), which is still consistent with the ISM density needed to "frustrate" its evolution. For the GPS galaxies presented in this paper, radio HI measurement are available only for PKS $0500+019$. The $r \simeq 84 \mathrm{pc}$ separated hot spots are covered by a HI column density of $\simeq 6 \times 10^{20} \mathrm{~cm}^{-2}$, therefore about one order of magnitude lower than observed in $\mathrm{X}$-rays. This implies that the X-ray absorber is located inside the hot-spots. If the jet has to drill its way through it before reaching the current separation, the expansion time is:

$t_{\mathrm{e}} \lesssim 7.3 \times 10^{4} L_{44}^{-0.5} \Omega_{10}^{0.5}$ years

(Scheuer 1974; Carvalho 1985) where $L_{44}$ is the (unknown) luminosity injected in the jet in units of $10^{44}$ and $\Omega_{10}$ is the jet opening angle in units of $10^{\circ}$. The "braking effect" of the $\mathrm{X}$-ray absorber is therefore negligible. Still, any residual particle density facing the expanding jet head is constrained only to be $n_{\mathrm{e}} \lesssim N_{\mathrm{H}} / r=20 \mathrm{~cm}^{-3}$. We know very little of the distribution of the ISM in the innermost kpc of this (and other) AGN and the ultimate fate of the jet in PKS 0500+019 is not known.

With typical intrinsic X-ray luminosities $\sim 10^{44} \mathrm{erg} \mathrm{s}^{-1}$, most GPS galaxies of our sample have a borderline luminosity between the galaxy and the quasar regimes. The X-ray spectral properties and luminosities suggest that they may belong to the long-sought class of obscured high-luminosity sources predicted by theoretical models of the Cosmic X-ray Background (CXB; Gilli et al. 2001). The cosmological evolution of GPS sources - which represents a sizable fraction of radio-selected extragalactic sources (O'Dea et al. 1998) - may

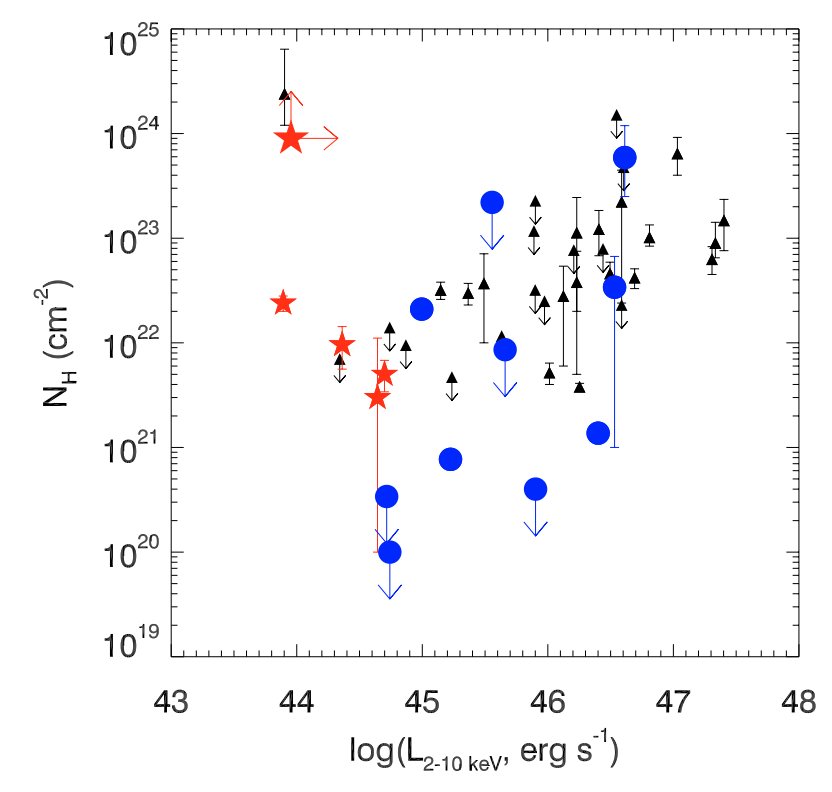

Fig. 8. $N_{\mathrm{H}}$ versus X-ray luminosity for GPS galaxies (stars) and GPS/CSS quasars (filled circles). The small triangles represent the radio-loud quasars observed by ASCA after Reeves \& Turner (2000).

have an impact on the CXB. In Fig. 8 we compare the $N_{\mathrm{H}}$ versus X-ray luminosity in the GPS galaxy sample with that measured in a sample of GPS/CSS quasars (S99; Siemiginowska et al. 2003; F05), and in the large compilation of spectra of radio-load quasars observed by ASCA after Reeves \& Turner (2000). In the latter, a correlation exists between the obscuring column density and the absorption-corrected $2-10 \mathrm{keV} \mathrm{lu}-$ minosity. Given the large fraction of upper limits on $N_{\mathrm{H}}$ in the sample, we have applied a $\operatorname{linear}$ fit $\log \left(N_{\mathrm{H}}\right)=a+b \times$ $\log \left(L_{\mathrm{X}}\right)$ by extending the regression method for left censored data described by Schmitt (1985) and Isobe et al. (1986). We performed a large number of least square fits on a set of MonteCarlo simulated data derived from the experimental points according to the following rules: a) each detection was substituted by a random Gaussian distribution, whose mean is the best-fit measurement and whose standard deviation is its statistical uncertainty; b) each upper limit $U$ was substituted by a random uniform distribution in the close interval $[0, U]$. The slope obtained with this method - albeit rather shallow - is larger than 0 at a confidence level greater than $5 \sigma: b_{\text {QSO }}=$ $(3.9 \pm 0.7) \times 10^{-3}$. The same fit applied on the GPS/CSS sample yields no significant evidence for a correlation, even if we include the GPS galaxies discussed in this paper: $b_{\mathrm{GPS} / \mathrm{CSS}}=$ $(-30 \pm 100) \times 10^{-3}$, although the large statistical uncertainties prevent us from drawing any firm conclusions. If this different behavior is confirmed on a larger sample, this may indicate that GPS/CSS quasars are a "mixed bag" of obscured/unbeamed and unobscured/beamed objects. Beamed structures in highluminosity quasars may create an unobscured line-of-sight by piercing their way through dense nuclear environment gas. In statistical terms, this would happen more rarely in lowerluminosity galaxies. This scenario cannot be investigated with the currently available sample, and would require good quality X-ray observations on a significantly larger, statistically unbiased sample of GPS sources. 


\section{Conclusions}

This paper investigates the apparent X-ray under-luminosity of GPS sources shown by ROSAT (Baker et al. 1995), by obtaining for the first time good quality X-ray spectra on a sizable sample thereof. The main conclusions of this study are:

- The GPS radio-to-X-ray Spectral Energy distributions are generally similar. The ratio between the $5 \mathrm{GHz}$ and the $5 \mathrm{keV}$ flux density is constrained within a factor $\simeq 15$. There is no unique reason for this residual scatter, which is likely to be due to a combination of X-ray obscuration and intrinsic luminosity effects. On the other hand, PKS 0941-080 is two orders of magnitude X-ray under-luminous with respect to the average of the other objects discussed in this paper. Whether this is due to high obscuration or intrinsic $\mathrm{X}$-ray weakness is impossible to tell with the current data. We therefore refrain from inferring any implications from this single outlier.

- The detection of rapid variability during the XMM-Newton observation of COINS J0029+3456 puts a constraint on the high-energy photon emitting region $\lesssim 10 \mu \mathrm{pc}$, supporting an origin at the base of the expanding jet (where radio synchrotron emission is likely to be self-absorbed) or in the accretion disk surrounding the active nucleus.

- The obscuring screen in PKS 0500+019 is located well within the radio hot spots, as suggested by the fact that the column density determined in X-rays is almost one order of magnitude higher than that estimated from radio measurements. The hot spots are therefore completely X-ray silent in PKS 0500+019. The lack of soft excess emission above the obscured X-ray emission in COINS J0029+3456 can be interpreted likewise.

- GPS galaxies exhibit a radio-to-X-ray luminosity ratio comparable to a control sample of "normal" radio galaxies. This indicates that it is unlikely that GPS galaxies are intrinsically X-ray weaker than their less compact counterparts.

- The X-ray obscuring gas column density distribution function for GPS galaxies is inconsistent at the $\gtrsim 98.7 \%$ level with the distribution function of a control sample of RGs. The main reason for this difference is the comparative paucity of X-ray unobscured $\left(N_{\mathrm{H}} \leq 10^{21.5} \mathrm{~cm}^{-2}\right)$ GPS galaxies.

- The average X-ray column density in our sample is consistent with that observed in the 4 HEG FR II galaxies for which measurements of this observable are available. This suggests that X-ray absorption in a GPS galaxy is mainly due to orientation with respect to an obscuring "torus".

- There is no compelling evidence for hot $\left(k T \sim 10^{6}-10^{7} \mathrm{~K}\right)$ gas from the X-ray spectra.

The above evidence support a scenario whereby GPS radio galaxies are "young" counterparts of large-scale FR II radio galaxies. $N_{\mathrm{H}} \sim 10^{22} \mathrm{~cm}^{-2}$ column densities in the innermost kpc around the active nucleus are unlikely to substantially affect the evolution of sub-kpc jet structures. However, tenuous ISM matter could have a much more important role in braking or smothering the full expansion of the radio structure. Signatures of this hot gas could be revealed by deep high-resolution X-ray Chandra observations.

Although the GPS sample presented in this paper represents the largest compilation ever published of X-ray measurements of this class of objects, the sample is small and admittedly far from complete or unbiased. On the contrary, it is clearly biased toward X-ray brightest (and/or therefore potentially less obscured) objects. Moreover, an accurate determination of the ionization state of the absorber is beyond the capability of the current high-resolution X-ray detectors for objects of X-ray flux $\sim 10^{-13} \mathrm{erg} \mathrm{cm}^{-1} \mathrm{~s}^{-1}$, and must await the next generation of X-ray missions. Questions such as the nature of the gaseous nuclear environment of the early evolutionary stages of AGN (di Matteo et al. 2005), or the feedback between the (recurrent? Siemiginowska et al. 2002, 2003) AGN activity and its environment, or surrounding clusters (Fabian et al. 2003; Siemiginowsha et al. 2005a) could be properly addressed by high quality spectroscopic X-ray measurements of a sizable sample of GPS sources. Nonetheless, the results presented in this paper open a so far unexplored window on the environmental conditions surrounding the origin of the radio power in the Universe. An extension of this study to a complete and unbiased sample of GPS galaxies is under way.

Acknowledgements. This paper is based on observations obtained with XMM-Newton, an ESA science mission with instruments and contributions directly funded by ESA Member States and the USA (NASA). This research has made use of data obtained through the High Energy Astrophysics Science Archive Research Center Online Service, provided by the NASA/Goddard Space Flight Center and of the NASA/IPAC Extragalactic Database (NED) which is operated by the Jet Propulsion Laboratory, California Institute of Technology, under contract with the National Aeronautics and Space Administration. This work was partly supported by NASA grants GO2-3148A and NNG04GF98G. A.S. acknowledges support by NAS8-39073. Careful reading of the manuscript by the anonymous referee is gratefully acknowledged.

\section{References}

Baker, J. C., Hunstead, R. W., \& Brinkmann, W. 1995, MNRAS, 277, 553

Barnes, J. E., \& Hernquist, L. 1996, ApJ, 471, 115

Baum, S. A., O’Dea, C. P., de Bruyn, A. G., \& Murphy, D. W. 1990, A\&A, 232, 19

Bennett, C. L., Halpurn, M., Hinshaw, G., et al. 2003, ApJS, 148, 1

Biretta, J. A., Schneider, D. P., \& Gunn, J. E. 1985, AJ, 90, 2508

Carilli, C. L., Menten, K. M., Reid, M. J., Rupen, M. P., \& Yun, M. S. 1998, ApJ, 494, 175

Carvalho, J. C. 1985, A\&A, 150, 129

Carvalho, J. C. 1994, A\&A, 292, 392

Carvalho, J. C. 1998, A\&A, 329, 845

Chiaberge, M., Capetti, A., \& Celotti, A. 1999, A\&A, 349, 77

Chiaberge, M., Duccio Macchetto, F., Sparks, W. B., et al. 2002, ApJ, 571,247

Condon, J. J., Cotton, W. D., Greisen, E. W., et al. 1998, AJ, 115, 1693

Dallacasa, D., Bondi, M., Alef, W., \& Mantovani, F. 1997, A\&A, 325, 943

de Vries, W. H., Barthel, P. D., \& Hes, R. 1985, A\&AS, 114, 259

de Vries, W. H., O’Dea, C. P., Perlman, E., et al. 1998, ApJ, 503, 138 
de Vries, W. H., O’Dea, C. P., Barthel, P. D., \& Thompson, D. J. 2000, A\&AS, 143, 181

De Young, D. S. 1993, ApJ, 402, 95

Dickey, J. M., \& Lockman, F. J. 1990, ARA\&A, 28, 215

di Matteo, T., Springel, V., \& Hernquist, L. 2005, Nature, 433, 604

Donato, D., Sambruna, R. M., \& Gliozzi, M. 2004, ApJ, 617, 915 (D04)

Edwards, P. G., \& Tringay, S. J. 2004, A\&A, 424, 91

Fabian, A. C., Sanders, J. S., Allen, S. W., et al. 2003, MNRAS, 344, L43

Fanti, C., Fanti, R., Dallacasa, D., et al. 1995, A\&A, 302, 317

Fiocchi, M. T., et al. 2005, ApJ, submitted (F05)

Fossati, G., Maraschi, L., Celotti, A., Comastri, A., \& Ghisellini, G. 1998, MNRAS, 299, 433

Fugmann, W., \& Meisenheimer, K. 1988, A\&AS, 76, 145

Gabriel, C., Denby, M., Fyfe, D. J., Hoar, J., \& Ibarra, A. 2003, in Astronomical Data Analysis Software and Systems XIII, ed. F. Ochsenbein, M. Allen, \& D. Egret (San Francisco: ASP), ASP Conf. Ser., 314, 759

Gehrels, N. 1986, ApJ, 303, 336

Gilli, R., Salvati, M., \& Hasinger, G. 2001, A\&A, 366, 407

Gopal-Krishna, \& Wiita, P. J. 1991, ApJ, 373, 325

Guainazzi, M., Oosterbroek, T., Antonelli, L. A., \& Matt, G. 2000, A\&A, 364, L80

Guainazzi, M., Siemiginowska, A., Rodriguez-Pascual, P., \& Stanghellini, C. 2004, A\&A, 421, 461

Guainazzi, M., Piconcelli, E., Jimenez-Bilón, E., \& Matt, G. 2005, A\&A, 429, L9

Isobe, T., Feigelson, E. D., \& Nelson, P. I. 1986, ApJ, 306, 490

Iwasawa, K., Allen, S., Fabian, A. C., Edge, A. C., \& Ettori, S. 1999, MNRAS, 306, 467

Jackson, N., \& Rawlings, S. 1997, MNRAS, 286, 241

Jiménez-Bailón, E., Piconcelli, E., Guainazzi, M., et al. 2005, A\&A, 435,449

Kadler, M., Kerp., J., Ros, E., et al. 2004, A\&A, 420, 467

Kollgaard, R. I., Feigelson, E. D., Laurent-Muehleisen, S. A., et al. 1995, ApJ, 449, 61

Kühr, H., Witzel, A., Pauliny-Toth, I. I. K., \& Nauber, U. 1981, A\&AS, 45, 367

Laing, R. A., Jenkins, C. R., Wall, J. V., \& Unger, S. W. 1994, in The First Stromlo Symposium: The Physics of Active Galactic Nuclei, ed. G. V. Bicknell, M. A. Dopita, \& P. A. Quinn, ASP Conf. Ser., 54,201

Ma, C., Arias, E. F., Eubanks, T. M., et al. 1998, AJ, 116, 516

Mazzarella, J. M., Bothun, G. D., \& Boroson, T. A. 1991, AJ, 101, 2034

Murgia, M. 2003, PASA, 20, 19

O'Dea, C. 1998, PASP, 107, 803

O'Dea, C., Stanghellini, C., Baum, S., \& Charlot, S. 1996, ApJ, 470, 806

O’Dea, C., de Vries, W. H., Worrall, D. M., Baum, S., \& Koekmoer, A. $2000, \mathrm{AJ}, 119,478$
Page, K. L., O’Brian, Reeves, J. N., \& Breeveld, A. A. 2003, MNRAS, 340, 1052

Peacock, J. A., Perryman, M. A. C., Longair, M. S., Gunn, J. E., \& Westphal, J. A. 1981, MNRAS, 194, 601

Perola, G. C., Matt, G., Cappi, M., et al. 2002, A\&A, 389, 202

Phillips, R. B., \& Mutel, R. L. 1982, MNRAS, 257, L19

Pihlström, Y. M., Conway, J. E., \& Vermeulen, R. C. 2003, A\&A, 404, 871

Polatidis, A. G., \& Conway, J. E. 2003, PASA, 20, 69

Readhead, A. C. S., Taylor, G. B., Pearson, T. J., \& Wilkinson, P. N. 1996, ApJ, 460, 634

Reeves, J. N., \& Turner, M. J. L. 2000, MNRAS, 316, 234

Reeves, J., Turner, M. J. L., Kii, T., \& Ohashi, T. 1997, MNRAS, 312 , L17

Risaliti, G. 2002, A\&A, 386, 379

Sambruna, R., Eracleous, M., \& Mushotzky, R. 1999, ApJ, 526, 60 (S99)

Satypal, S., Sambruna, R. M., \& Dudik, R. P. 2004, A\&A, 414, 8235

Scheuer, P. A. G. 1974, MNRAS, 166, 513

Schmitt, J. H. M. M. 1985, A\&A, 293, 178

Siemiginowska, A., Bechtold, J., Aldcroft, T. L., et al. 2002, ApJ, 570, 543

Siemiginowska, A., Aldcroft, T. L., Bechtold, J., et al. 2003, PASA, 20,113

Siemiginowska, A., Cheung, C. C., LaMassa, S., et al. 2005a, ApJ, submitted

Siemiginowska, A., et al. 2005b, ApJ, submitted

Snellen, I. A. G., Bremer, M. N., Schillizzi, R. T., et al. 1996, MNRAS, 279,1294

Stanghellini, C., O’Dea, C. P., Baum, S. A., \& Laurikainen, E. 1993, ApJS, 88, 1

Stanghellini, C., O'Dea, C. P., Baum, S. A., et al. 1997, A\&A, 325, 953

Stanghellini, C., O’Dea, C. P., Dallacasa, D., et al. 1998, A\&AS, 131, 303

Stanghellini, C., Dallacasa, D., O’Dea, C. P., et al. 2001, A\&A, 377, 377 (erratum: 379, 870)

Stickel, M., Rieke, M. J., Rieke, G. H., \& Kuehr, H. 1996, A\&A, 306, 49

Strüder, L., Briel, U., Dannerl, K., et al. 2001, A\&A, 365, L18

Taniguchi, Y., \& Wada, K. 1996, ApJ, 469, 581

Tingay, S. J., Edwards, P. G., \& Tzioumis, A. K. 2003, MNRAS, 346, 327

Turner, M. J. L., Abbey, A., Arnaud, M., et al. 2001, A\&A, 365, L27

Urry, C. M., \& Padovani, P. 1995, PASP, 107, 803

Varano, S., Chiaberge, M., Macchetto, F. D., \& Capetti, A. 2004, A\&A, 428, 401

Voges, W., Aschenbach, B., Boller, Th., et al. 2000, IAUC 7432

Weisskopf, M. C., Brinkman, B., Canizares, C., et al. 2002, PASP, 114, 1

Zensus, J. A., Ros, E., Kellermann, K. I., et al. 2002, AJ, 124, 662 